\title{
Giant Intracranial Aneurysms at 7T MRI
}

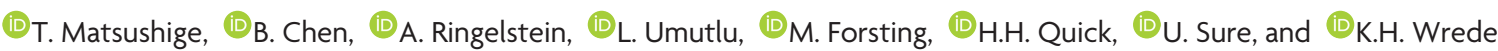

\begin{abstract}
SUMMARY: Giant intracranial aneurysms are rare vascular pathologies associated with high morbidity and mortality. The purpose of this in vivo study was to assess giant intracranial aneurysms and their wall microstructure by 7T MR imaging, previously only visualized in histopathologic examinations. Seven giant intracranial aneurysms were evaluated, and 2 aneurysms were available for histopathologic examination. Six of 7 (85.7\%) showed intraluminal thrombus of various sizes. Aneurysm walls were depicted as hypointense in TOF-MRA and SWI sequences with excellent contrast ratios to adjacent brain parenchyma (range, 0.01-0.60 and 0.58-0.96, respectively). The triple-layered microstructure of the aneurysm walls was visualized in all aneurysms in TOF-MRA and SWI. This could be related to iron deposition in the wall, similar to the findings in 2 available histopathologic specimens. In vivo 7T TOF-MRA and SWI can delineate the aneurysm wall and the triple-layered wall microstructure in giant intracranial aneurysms.
\end{abstract}

ABBREVIATIONS: $G A=$ giant intracranial aneurysm; $\mathrm{SEM}=$ standard error of the mean

ntracranial saccular aneurysms are defined as saccular dilations of cerebral arterial vessels. The overall prevalence of unruptured intracranial aneurysms is estimated between $0.5 \%$ and $7 \%$ of the general population with a $0.5 \%-1.1 \%$ rupture risk per year on average. ${ }^{1}$ Considering the natural history, giant intracranial aneurysms (GAs, defined as $>25 \mathrm{~mm}$ in maximum diameter) belong to a rare subgroup (approximately $0.5 \%$ of all unruptured cerebral aneurysms), which are highly prone to rupture. ${ }^{2,3}$

The pathophysiology of the growth in GAs is considered to differ from that of non-GAs. The presence of intraluminal thrombus formation associated with repeated intramural hemorrhage and neovascularization in the wall seems to play an important role in GA

Received June 23, 2015; accepted after revision August 20.

From the Department of Neurosurgery (T.M., B.C., U.S., K.H.W.), University Hospital Essen, University Duisburg-Essen, Essen, Germany; Department of Neurosurgery (T.M.), Graduate School of Biomedical and Health Sciences, Hiroshima University, Hiroshima, Japan; Erwin L. Hahn Institute for Magnetic Resonance Imaging (T.M., B.C., L.U., H.H.Q., K.H.W.), University Duisburg-Essen, Essen, Germany; and Department of Diagnostic and Interventional Radiology and Neuroradiology (A.R., L.U., M.F.) and High Field and Hybrid MR Imaging (H.H.Q.), University Hospital Essen, Essen, Germany

This work was supported by the University Duisburg Essen Interne Forschungsförderung Essen grant to Karsten $\mathrm{H}$. Wrede.

Paper previously presented at: Annual Meeting of the International Society for Magnetic Resonance in Medicine, May 30 -June 5, 2015; Toronto, Ontario, Canada; and as a poster at: Annual Meeting of the German Society of Neurosurgery, June 7-10 2015; Karlsruhe, Germany.

Please address correspondence to Toshinori Matsushige, MD, University Hospital Essen, Department of Neurosurgery, Hufelandstr 55, 45147 Essen, Germany; e-mail address: Toshinori.Matsushige@uk-essen.de

http://dx.doi.org/10.3174/ajnr.A4569 growth. ${ }^{4,5}$ Prior histopathologic studies of intracranial aneurysms have shown inflammation and associated iron deposits in the wall that correlated with growth and rupture of the aneurysm. ${ }^{6,7}$ Previous MR imaging studies have revealed important morphologic features in these aneurysms, which are only partially assessable by DSA. ${ }^{8,9}$ Better delineation and characterization of GAs may help to improve our comprehension of the distinct pathophysiology of this rare aneurysm subgroup. The purpose of this study was to assess GAs and their wall microstructure by 7T MR imaging, previously only visualized in histopathologic examinations.

\section{MATERIALS AND METHODS}

The study was approved by the authorized ethics committee of the local university, and all patients provided written consent before the examination. Inclusion criteria were the following: 1) patients with a GA previously diagnosed via DSA and conventional $1.5 \mathrm{~T}$ or 3T MR imaging, 2) 18 years of age or older, and 3) able to give informed consent. Exclusion criteria were the following: 1) the presence of a cardiac pacemaker or any other electronic implants, 2) pregnancy or breastfeeding, or 3) claustrophobia. Patients were recruited from January 2011 to December 2014 and included 4 men and 3 women with an average age of 66 years (range, 50-80 years).

\section{High-Resolution 7T MR Imaging}

Seven patients with GAs were evaluated by using a 7T whole-body MR imaging system (Magnetom 7T; Siemens, Erlangen, Germany) equipped with a 32-channel Tx/Rx head coil (Nova Med- 
ical, Wilmington, Massachusetts). The system is equipped with a gradient system providing $45-\mathrm{mT} / \mathrm{m}$ maximum amplitude and a slew rate of $200 \mathrm{mT} / \mathrm{m} / \mathrm{ms}$. Applied sequences included TOFMRA $^{10,11}$ and MPRAGE ${ }^{12}$ from the standard 7T vascular protocol and SWI for detecting iron deposition. Detailed parameters for all sequences are summarized in Table 1.

\section{Image Analysis}

Before the evaluation, image data for all individual cases were coregistered between sequences by using the FMRIB Linear Image Registration Tool (FLIRT; http://www.fmrib.ox.ac.uk). Image evaluation was performed by using the FSLView tool (FMRIB Software Library, Version 5.0; http://fsl.fmrib.ox.ac.uk/fsl/fslview/) by 2 raters in a consensus reading. Assessed features were the following: aneurysm and parent artery diameter, wall thickness, signal intensity of the aneurysm wall, intraluminal thrombus, intraluminal flow, and surrounding brain tissue. The ROIs were placed in the wall of the dome farthest away from the aneurysm base, including 4 representative voxels $(0.44 \times 0.44 \mathrm{~mm})$ of each structure. The ROIs in the tissues surrounding the wall amounted to 16 voxels $(0.88 \times 0.88 \mathrm{~mm})$. Absolute values for contrast ratios of aneurysm wall layers and adjacent tissues were calculated as follows:

$$
\left|\frac{\left(\text { Intensity }_{\mathrm{a}}-\text { Intensity }_{\mathrm{b}}\right)}{\left(\text { Intensity }_{\mathrm{a}}+\text { Intensity }_{\mathrm{b}}\right)}\right| .
$$

For every mean value, the standard error of mean (SEM, $\sigma / \sqrt{n})$ was calculated as an estimate of the population mean.

\section{Histopathologic Examination}

To surgically expose the aneurysm neck, partial resection of the wall and thrombus was mandatory in 2 thrombosed MCA aneurysms preoperatively scanned with 7T MR imaging. Aneurysm domes were successfully resected parallel to the parent artery. Histopathologic sections were prepared from areas corresponding to MR imaging ROIs with 5 - $\mu \mathrm{m}$ thickness and were stained by using H\&E, Van Gieson elastic, and Prussian blue.

\section{RESULTS}

All patients were examined without any adverse events, and all MR imaging sequences were successfully acquired. Table 2 summarizes basic demographic data for all patients and major anatomic features of the aneurysms. The mean diameters of aneurysms and parent arteries were $29.8 \mathrm{~mm}$ (SEM, 1.9; range, 25.1-37.9 mm) and $3.3 \mathrm{~mm}$ (SE, 0.4; range, 1.7-5.3 mm), respectively. All aneurysms except 1 were surrounded by brain parenchyma, and 4 aneurysms were accompanied by brain edema. The aneurysm arising from the cavernous portion of the ICA (subject 2) extended into the intracranial space with the cranial part of the dome. Figure 1 illustrates specific features of aneurysm delineation for all 7 cases in TOF-MRA, MPRAGE, magnitude imaging, and SWI.

\section{Thickness of the Aneurysm Wall}

The mean aneurysm wall thickness was $0.96 \mathrm{~mm}$ (SEM, 0.1; range, $0.73-1.39 \mathrm{~mm}$ ) in the TOF-MRA sequence, $1.08 \mathrm{~mm}$ (SEM, 0.11; range, $0.79-1.55 \mathrm{~mm}$ ) in MPRAGE, $1.34 \mathrm{~mm}$ (SEM, 0.08; range, $1.11-1.67 \mathrm{~mm}$ ) in magnitude images, and $1.45 \mathrm{~mm}$ (SEM, 0.09; range, $1.22-1.86 \mathrm{~mm}$ ) in SWI. The thicknesses in histopathologic sections of aneurysm walls near the fundus were $0.83-0.95 \mathrm{~mm}$ (subject 3) and 0.50-0.57 mm (subject 5). The thickness measured in histopathologic sections corresponded best with thickness measured in the TOF-MRA sequence for both patients who underwent surgery. Measured thicknesses of aneurysm walls are summarized in Fig 2.

\section{Signal Intensity of the Aneurysm Wall}

Aneurysm walls were depicted as hypointense on TOF-MRA and strongly hypointense on magnitude images and SWI compared with adjacent structures. In MPRAGE, walls were delineated with a heterogeneous mostly hypointense signal. All aneurysms demonstrated a characteristic feature, showing a high contrast ratio of the wall to adjacent brain parenchyma. Signal intensities of intraluminal thrombus were heterogeneous between and within aneurysms. How-

Table 2: Patient demographics and anatomic characteristics for all examined aneurysms

\begin{tabular}{|c|c|c|c|c|c|c|c|c|}
\hline Nr. & $\begin{array}{l}\text { Age } \\
\text { (yr) }\end{array}$ & Sex & Location & Thrombosis & $\begin{array}{c}\text { Maximum } \\
\text { Diameter }(\mathrm{mm})\end{array}$ & $\begin{array}{l}\text { Aneurysm Neck } \\
\text { Diameter (mm) }\end{array}$ & $\begin{array}{c}\text { Parent Vessel } \\
\text { Diameter }(\mathrm{mm})\end{array}$ & Treatment \\
\hline 1 & 50 & $\mathrm{~F}$ & ICA & Partially & 25.06 & 4.38 & 3.85 & Endovascular $^{a}$ \\
\hline 2 & 56 & $\mathrm{~F}$ & ICA (cavernous) & Partially & 37.87 & 7.95 & 3.43 & Endovascular ${ }^{b}$ \\
\hline 3 & 75 & $M$ & MCA & Partially & 35.78 & 6.79 & 3.04 & Clipping $^{c}$ \\
\hline 4 & 80 & $M$ & MCA & Completely & 28.99 & 7.31 & 1.70 & Observation \\
\hline 5 & 61 & $\mathrm{~F}$ & MCA & Partially & 25.95 & 5.60 & 2.46 & Clipping \\
\hline 6 & 69 & $M$ & Basilar artery & Completely & 27.21 & 22.10 & 3.57 & Observation \\
\hline 7 & 71 & $M$ & Basilar artery & None & 27.75 & 25.86 & 5.31 & Observation \\
\hline
\end{tabular}

Note:-Nr. indicates patients in chronological order.

${ }^{a}$ Coiling with a stent.

${ }^{b}$ Parent vessel occlusion.

'Subject with histopathologic assessment. 




FIG 1. Delineation of giant intracranial aneurysms by 7T MR imaging for all 7 patients by TOF-MRA $(A)$, MPRAGE (B), magnitude imaging (C), and SWI (D). Specific structures are marked identically in all subfigures. Arrows indicate the aneurysm wall; white asterisks, intraluminal thrombus; black asterisks, flow lumen; and double daggers, brain parenchyma. Aneurysm walls show hypointense signal in TOF-MRA, magnitude imaging, and SWI and heterogeneous signal intensity in MPRAGE.

ever, thrombus areas adjacent to the aneurysm wall were generally hyperintense in comparison with the wall. Contrast between intraluminal thrombus and brain parenchyma was low for all MR imaging sequences. Partially thrombosed aneurysms (subjects 1,3, and 5) showed heterogeneous contrast between thrombus and intraluminal flow. Details of contrast ratios are summarized in Table 3.

In TOF-MRA and SWI, aneurysm walls adjacent to brain parenchyma were depicted partially with a distinct triple-layered microstructure showing a hyperintense signal in the middle layer 
surrounded by hypointense outer and inner layers. Mean contrast ratios between the middle layer and outer/inner layers were high (0.27-0.84) with better contrast in SWI than in TOF (Table 4). In 2 available histopathologic specimens, the hyperintense layer corresponded to the tunica media. There was no iron deposition within this part of the wall in the Prussian blue stain, while adventitia and smooth muscle layer adjacent to intraluminal thrombus showed strong iron uptake (Fig 3).

\section{DISCUSSION}

To our knowledge, this is the first in vivo study investigating GAs using ultra-high-field 7T MR imaging. Because most patients with GAs present with urgent clinical symptoms, ${ }^{5}$ for most of these cases, 7T MR imaging examinations remain inaccessible. Considering the scarce opportunity to recruit patients with this extremely rare vascular disease, this study of GAs can help to further understand the complex pathophysiology of aneurysm formation, growth, and rupture.

In previously published in vivo aneurysm wall imaging studies, depiction of microstructures within aneurysm walls was limited by spatial resolution, ${ }^{13-15}$ because wall thicknesses ranged from 20 to $500 \mu \mathrm{m} .^{16,17}$ To date, there is only 1 report on intracranial vessel wall microstructures delineated with 7T MR imaging using an ultra-high ex vivo $0.11 \times 0.11 \mathrm{~mm}^{2}$ resolution. ${ }^{18}$ The study showed excellent correlation with the histopathologic findings. However, the presented ex vivo protocol took 40.5 hours per specimen and is therefore unsuitable for clinical in vivo application. In view of the previously published data, spatial resolution has to be well below aneurysm wall thickness to be capable of demonstrating microstructures within the wall. The spatial resolution used in our study was 5.7-13.2 times higher compared with previous in vivo aneurysm wall studies, ${ }^{10,11,13-15}$ which was sufficient to visualize the microstructures in vivo for the first time.

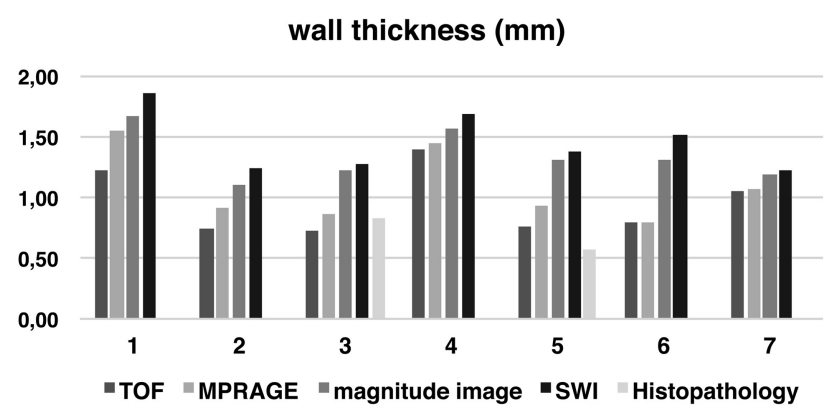

FIG 2. Thickness of aneurysm walls is presented for all MR imaging sequences and histopathology (only available for cases 3 and 5). In every subject, aneurysm wall thickness was larger on magnitude images and SWI compared with TOF-MRA and MPRAGE. Histopathologic measurements correlated best with TOF-MRA measurements in all subjects.
Histopathology revealed a distinct pattern of iron deposition within the aneurysm wall, showing a homogeneous distribution in the adventitia and smooth muscle layer adjacent to intraluminal thrombus, sparing the tunica media. Honkanen et $\mathrm{al}^{19}$ characterized the aneurysm wall in an ex vivo study setup comparing 4.7T MR imaging and histopathology, showing the capability of a $2^{*}$ sequence to detect iron-loaded macrophages depicting the degenerated aneurysm walls as hypointense structures. In the present study, the ultra-high spatial resolution in TOF-MRA and SWI could depict iron-related signal decay in voxels within the aneurysm wall as a typical triple-layered microstructure, which correlates with the histopathologic findings. Due to the lower spatial resolution of MPRAGE, partial volume effects of surrounding inner and outer aneurysm wall layers blurred the tunica media signal. Thus, MPRAGE was not able to depict the distinct triple-layered wall structure, showing only a diffuse hypointense signal.

The surrounding brain parenchyma and intraluminal thrombus, often seen in GAs, provide additional image contrast and therefore help to differentiate the aneurysm wall from its surrounding structures. In some cases, heterogeneous signal in parts of the intraluminal thrombus led to local low-contrast areas around the aneurysm wall, making differentiation slightly more difficult. The inhomogeneous iron deposition caused by different ages of thrombus layers ${ }^{20}$ is presumably associated with the heterogeneous signal intensity in some thrombus. As expected, the local B0 field inhomogeneity due to the deposited iron caused stronger signal alterations in SWI compared with TOF-MRA and MPRAGE. These susceptibility effects are amplified at 7T compared with lower magnetic field strengths. Because of strong blooming effects in SWI at ultra-high magnetic field strengths, aneurysm wall thickness was overestimated approximately 1.5fold compared with TOF-MRA, which showed excellent correlation with histopathologic measurements.

It is debatable whether the triple-layered microstructures are fragile or represent a stable region of the aneurysm wall and to what extent iron accumulation derives from infiltrating macrophages. Considering previously published data from histopathologic and clinical studies and the present high-resolution MR imaging findings, the triple-layered microstructures are likely to reflect the fragility of the aneurysm wall. The grade of iron depo-

Table 4: Contrast ratios of aneurysm wall layers

\begin{tabular}{lcc}
\hline & $\begin{array}{c}\text { WLCR (Outer) (mean) } \\
\text { (SEM, range) }\end{array}$ & $\begin{array}{c}\text { WLCR (Inner) (mean) } \\
\text { (SEM, range) }\end{array}$ \\
\hline TOF & $0.39(0.04,0.24-0.59)$ & $0.27(0.04,0.15-0.49)$ \\
MPRAGE & NA & NA \\
Magnitude image & $0.49(0.10,0.25-1.00)$ & $0.33(0.11,0.14-1.00)$ \\
SWI & $0.84(0.06,0.52-1.00)$ & $0.71(0.08,0.31-1.00)$ \\
\hline
\end{tabular}

Note:-WLCR (outer) indicates wall middle layer to outer layer; WLCR (inner), wall middle layer to inner layer; NA, not applicable.

Table 3: Contrast ratios of aneurysm wall and intraluminal thrombus to adjacent structures

\begin{tabular}{lcccc}
\hline & WBCR (mean) (SEM, range) & WTCR (mean) (SEM, range) & TBCR (mean) (SEM, range) & TLCR (mean) (SEM, range) \\
\hline TOF & $0.41(0.08,0.01-0.60)$ & $0.33(0.10,0.03-0.58)$ & $0.15(0.10,0.23-0.57)$ & $0.31(0.11,0.16-0.62)$ \\
MPRAGE & $0.20(0.06,0.06-0.49)$ & $0.36(0.09,0.07-0.57)$ & $0.17(0.13,0.25-0.56)$ & $0.23(0.07,0.03-0.38)$ \\
Magnitude image & $0.67(0.05,0.44-0.83)$ & $0.54(0.15,0.08-0.82)$ & $0.26(0.10,0.06-0.75)$ & $0.30(0.16,0.02-0.84)$ \\
SWI & $0.84(0.05,0.58-0.96)$ & $0.70(0.15,0.13-0.93)$ & $0.12(0.16,0.19-0.95)$ & $0.34(0.18,0.07-0.97)$ \\
\hline
\end{tabular}

Note:- -WBCR indicates aneurysm wall to brain parenchyma; WTCR, aneurysm wall to intraluminal thrombus; TBCR, intraluminal thrombus to brain parenchyma; TLCR, intraluminal thrombus to intraluminal flow. 

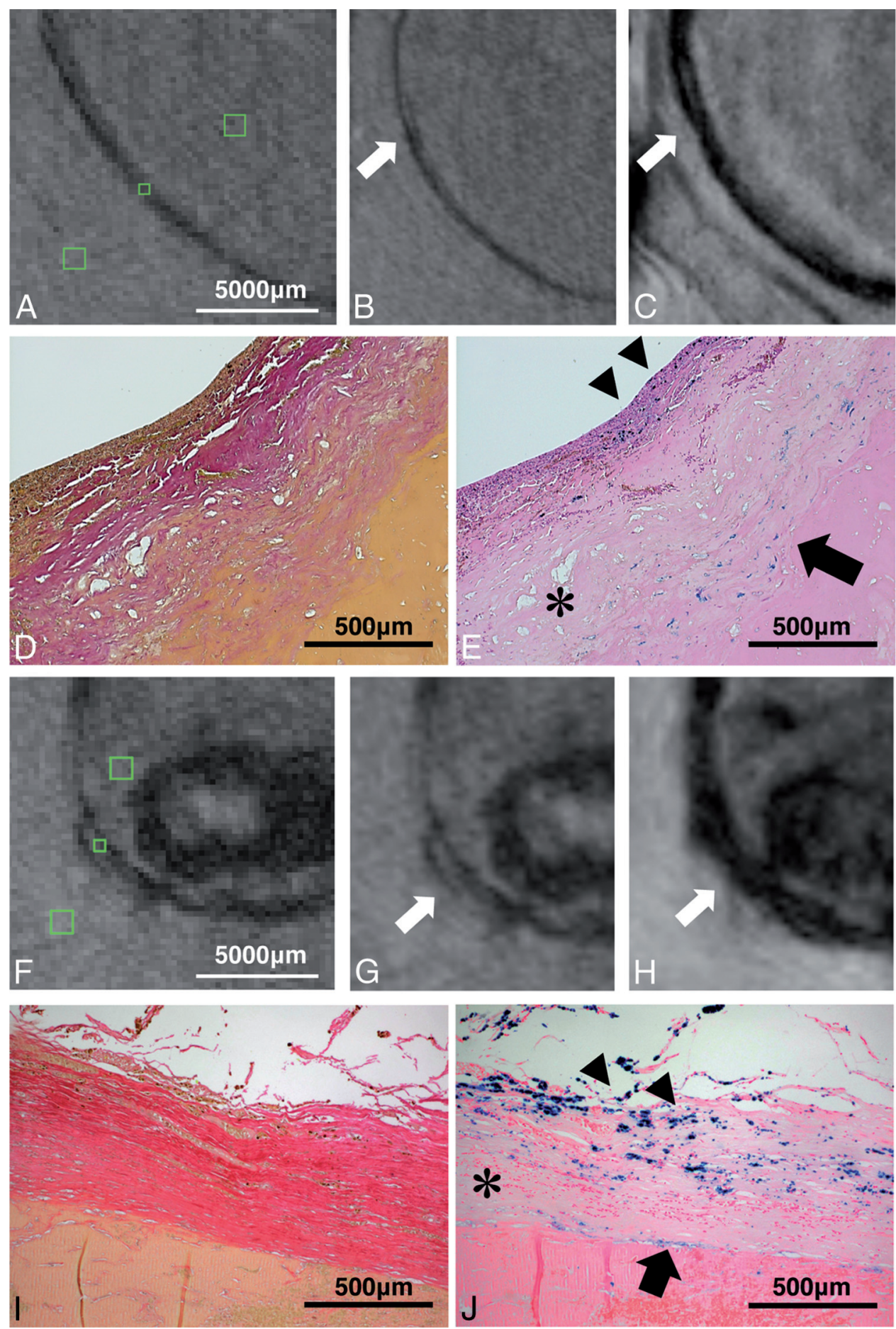

FIG 3. Histopathologic images and MR imaging of both partially resected aneurysms are illustrated. There is an excellent correspondence between hypointense signal in TOF-MRA and SWI and iron deposition in the aneurysm wall in histopathologic sections (subject 3: A-E; subject 5: $F-J)$. Green squares on TOF-MRA (without zoom interpolation) indicate ROls in the aneurysm wall and surrounding structures (brain parenchyma and intraluminal thrombus) ( $A$ and $F$ ). Magnified images depict the hypointense aneurysm wall in TOF-MRA ( $B$ and $G$ ) and the strongly hypointense aneurysm wall in SWI ( $C$ and $H$ ). Aneurysm walls partially show the triple-layered microstructure (hyperintense layer in the middle) in both sequences (arrows). Histopathology shows aneurysm walls with Van Gieson elastic staining (original magnification $\times 50)(D$ and $I$ ) and iron deposition in inner smooth muscle (arrowheads) and adventitial layer (arrow) in Prussian blue staining (original magnification $\times 50)(E$ and $/)$. Note the decellularized layer in the middle layer without iron deposition (asterisks).

sition, likely linked to inflammation, is detectable and can be quantified by using high-field MR imaging. This information might therefore serve as a future follow-up marker and help in deciding the treatment timing and technique.

\section{Limitations}

The present series consisted of a relatively small number of 7 patients with GAs. However, considering the extremely low incidence of this rare pathology, the possibility of recruiting a consid- 
erably larger number of patients within a reasonable time is limited. More than half of our patients presenting with giant aneurysms during these 3 years were unsuitable for scanning at $7 \mathrm{~T}$ MR imaging because of numerous contraindications. On the one hand, heterogeneous sizes and locations of scanned aneurysms seem to be a limitation of the study; on the other hand, identical signal characteristics of aneurysm walls could be shown in various locations and therefore indicate these signal characteristics as a general feature of GAs. Wall imaging in this study was focused on the fundus of the aneurysm. Walls of intracranial aneurysms are highly heterogeneous, ${ }^{15,21}$ making it difficult to characterize the complete aneurysm wall by using MR imaging and histopathology, when only parts of the aneurysm wall are investigated by both methods. A future ex vivo or in vivo study might overcome this limitation by 3D aligning of MR imaging and histopathologic images, allowing exact quantification of the complete aneurysm wall. Finally, susceptibility artifacts from adjacent air-filled sinuses can strongly impair aneurysm delineation close to the skull base, especially in SWI, making it difficult to visualize large parts of the aneurysm wall and therefore reducing applicability of 7T MRA as a follow-up marker.

\section{CONCLUSIONS}

High-resolution in vivo 7T SWI and TOF-MRA imaging can delineate the triple-layered wall microstructure in giant intracranial aneurysms previously only depicted by using ex vivo MR imaging or histopathologic examinations. Quantification of iron deposition in the aneurysm wall might serve as a future follow-up marker and help in deciding the treatment timing and technique.

\section{ACKNOWLEDGMENTS}

The authors thank Lena C. Schäfer (RT) for performing all the 7T examinations.

Disclosures: Bixia Chen—RELATED: Grant: Interne Forschungsförderung Essen, Comments: The Interne Forschungsförderung Essen grant is a scientific grant given by the University Duisburg-Essen; UNRELATED: Travel/Accommodations/Meeting Expenses Unrelated to Activities Listed: German Academic Exchange Service travel grant to the International Society for Magnetic Resonance in Medicine Annual Meeting and Exhibition. Karsten H. Wrede-RELATED: Grant: Interne Forschungsförderung Essen, Comments: The grant is a scientific one given by the University Duisburg-Essen.

\section{REFERENCES}

1. Brown RD Jr, Broderick JP. Unruptured intracranial aneurysms: epidemiology, natural history, management options, and familial screening. Lancet Neurol 2014;13:393-404 CrossRef Medline

2. Wiebers DO, Whisnant JP, Huston J 3rd, et al; International Study of Unruptured Intracranial Aneurysms Investigators. Intracranial aneurysms: natural history, clinical outcome, and risks of surgical and endovascular treatment. Lancet 2003;362:103-10 CrossRef Medline

3. Morita A, Kirino T, Hashi K, et al; UCAS Japan Investigators. The natural course of unruptured cerebral aneurysms in a Japanese cohort. N Engl J Med 2012;366:2474-82 CrossRef Medline
4. Barth A, de Tribolet N. Growth of small saccular aneurysms to giant aneurysms: presentation of three cases. Surg Neurol 1994;41:277-80 CrossRef Medline

5. dos Santos ML, Spotti AR, dos Santos RM, et al. Giant intracranial aneurysms: morphology and clinical presentation. Neurosurg Rev 2013;36:117-22; discussion 122 CrossRef Medline

6. Kataoka K, Taneda M, Asai T, et al. Structural fragility and inflammatory response of ruptured cerebral aneurysms: a comparative study between ruptured and unruptured cerebral aneurysms. Stroke 1999;30:1396-401 CrossRef Medline

7. Frösen J, Piippo A, Paetau A, et al. Remodeling of saccular cerebral artery aneurysm wall is associated with rupture: histological analysis of 24 unruptured and 42 ruptured cases. Stroke 2004;35:2287-93 CrossRef Medline

8. Roccatagliata L, Guédin P, Condette-Auliac S, et al. Partially thrombosed intracranial aneurysms: symptoms, evolution, and therapeutic management. Acta Neurochir 2010;152:2133-42 CrossRef Medline

9. Martin AJ, Hetts SW, Dillon WP, et al. MR imaging of partially thrombosed cerebral aneurysms: characteristics and evolution. AJNR Am J Neuroradiol 2011;32:346-51 CrossRef Medline

10. Johst S, Wrede KH, Ladd ME, et al. Time-of-flight magnetic resonance angiography at $7 \mathrm{~T}$ using venous saturation pulses with reduced flip angles. Invest Radiol 2012;47:445-50 CrossRef Medline

11. Wrede KH, Johst S, Dammann P, et al. Improved cerebral time-offlight magnetic resonance angiography at 7 Tesla: feasibility study and preliminary results using optimized venous saturation pulses. PLoS One 2014;9:e106697 CrossRef Medline

12. Wrede KH, Johst S, Dammann P, et al. Caudal image contrast inversion in MPRAGE at 7 Tesla: problem and solution. Acad Radiol 2012;19:172-78 CrossRef Medline

13. Park JK, Lee CS, Sim KB, et al. Imaging of the walls of saccular cerebral aneurysms with double inversion recovery black-blood sequence. J Magn Reson Imaging 2009;30:1179-83 CrossRef Medline

14. Kim TW, Choi HS, Koo J, et al. Intramural hematoma detection by susceptibility-weighted imaging in intracranial vertebral artery dissection. Cerebrovasc Dis 2013;36:292-98 CrossRef Medline

15. Kleinloog R, Korkmaz E, Zwanenburg JJ, et al. Visualization of the aneurysm wall: a 7.0-Tesla magnetic resonance imaging study. $\mathrm{Neu}$ rosurgery 2014;75:614-22; discussion 622 CrossRef Medline

16. Steiger HJ, Aaslid R, Keller S, et al. Strength, elasticity and viscoelastic properties of cerebral aneurysms. Heart Vessels 1989;5:41-46 CrossRef Medline

17. Boussel L, Wintermark M, Martin A, et al. Monitoring serial change in the lumen and outer wall of vertebrobasilar aneurysms. AJNR Am J Neuroradiol 2008;29:259-64 CrossRef Medline

18. Dieleman N, van der Kolk AG, Zwanenburg JJ, et al. Imaging intracranial vessel wall pathology with magnetic resonance imaging: current prospects and future directions. Circulation 2014;130:192201 CrossRef Medline

19. Honkanen P, Frösen JK, Abo-Ramadan U, et al. Visualization of luminal thrombosis and mural iron accumulation in giant aneurysms with ex vivo 4.7T magnetic resonance imaging. Surg Neurol Int 2014;5:74 CrossRef Medline

20. Corti R, Osende JI, Fayad ZA, et al. In vivo noninvasive detection and age definition of arterial thrombus by MRI. J Am Coll Cardiol 2002; 39:1366-73 CrossRef Medline

21. Kadasi LM, Dent WC, Malek AM. Cerebral aneurysm wall thickness analysis using intraoperative microscopy: effect of size and gender on thin translucent regions. J Neurointerv Surg 2013;5:201-06 CrossRef Medline 